TAIWANESE JOURNAL OF MATHEMATICS

Vol. 6, No. 3, pp. 369-382, September 2002

This paper is available online at http://www.math.nthu.edu.tw/tjm/

\title{
REMARKS ON IMPLICIT VECTOR VARIATIONAL INEQUALITIES ${ }^{1}$
}

\author{
Sangho Kum ${ }^{2}$ and Gue Myung Lee
}

\begin{abstract}
In this paper, as a continuation of the authors' work, the existence of solutions of IVVI for noncompact valued multifunctions is provided under generalized pseudomonotonicity assumption. This generalizes Konnov and Yao's results [6, Theorems 3.1 and 4.1]. Also another IVVI without the generalized pseudomonotonicity assumption is proposed and the existence of solutions of the IVVI is proved by using a selection theorem. This IVVI can be regarded as an extension of the VVI studied in Lee et al. [10].
\end{abstract}

\section{INTRODUCTION}

Since Giannessi [4] first introduced a vector variational inequality (shortly, VVI) in a finite dimensional Euclidean space, many authors have intensively studied VVI and its various extensions $[1,3,8-13,16,18]$ (see also the references therein) in abstract spaces. Systematic treaments on generalized VVI can be found in Konnov and Yao [6], and Lin et al. [14]. Besides, several authors have investigated relationships between VVI and vector optimization problems under some convexity or generalized convexity assumptions. Lee et al. [11] showed that VVI can be an efficient tool for studying vector optimization problems.

On the other hand, recently, Lee and Kum [13] proposed implicit vector variational inequalities (IVVI) to develope a general scheme dealing with seemingly diverse VVIs. They derived sufficient conditions for an IVVI of compact valued multifunctions to have solutions under generalized weak pseudomonotonicity assumptions and the Hausdorff topological vector space setting. In relation to IVVI,

Received August 30, 2000; revised February 15, 2001.

Communicated by M. H. Shih.

2000 Mathematics Subject Classification: 49J40, 47H10, 47H05, 54C60.

Key words and phrases: Vector variational inequality, $C$-pseudomonotone, $v$-hemicontinuous, FanBrowder fixed point Theorem, Selection theorem.

${ }^{1}$ This research was supported by Korea Research Foundation Grant No. KRF-99-042-D00018

${ }^{2}$ The author to whom the correspondence is directed 
Ansari and Yao [2] gave some interesting existence results for the strong solutions of IVVI which seems to be a proper generalization of solutions of generalized VVI.

In this paper, as a continuation of the authors' work [13], the existence of solutions of IVVI for noncompact valued multifunctions is provided under generalized pseudomonotonicity assumption. This generalizes Konnov and Yao's results [6, Theorems 3.1 and 4.1]. For this purpose, we slightly modify the definition of generalized hemicontinuity of a multifunction $T$ with respect to a general function $\psi$. Also another IVVI without the generalized pseudomonotonicity assumption is suggested and the existence of solutions of the IVVI is proved by using a selection theorem. This IVVI can be regarded as an extension of the VVI studied in Lee et al. [10].

We use a fixed point theorem due to Park [15] as a basic tool to establish our results.

\section{Preliminaries}

Let $E$ be a real Hausdorff topological vector space, $X$ a nonempty convex subset of $E, F$ another real Hausdorff topological vector space. A nonempty subset $P$ of $E$ is called a convex cone if

$$
\lambda P \subset P, \quad \text { for all } \quad \lambda \geq 0 \text { and } P+P=P .
$$

Let $C: X \rightarrow 2^{F}$ be a multifunction such that for each $x \in X, C x$ is a convex cone in $F$ with int $C x \neq \emptyset$ and $C x \neq F$. We denote

$$
P:=\bigcap_{x \in X} C x
$$

Let $L(E, F)$ be the space of all continuous linear mappings from $E$ to $F, \psi$ : $L(E, F) \times X \times X \rightarrow F$ a function, $T: X \rightarrow 2^{L(E, F)}$ a multifunction and $G$ : $X \rightarrow 2^{X}$ a multifuntion. In this paper, our discussion is restricted to the following (IVVI) and (IVVI)':

(IVVI) Find $\bar{x} \in X$ such that for each $y \in X$, there exists $s \in T \bar{x}$ satisfying

$$
\psi(s, \bar{x}, y) \notin-\operatorname{int} C \bar{x} .
$$

(IVVI) ${ }^{\prime}$ Find $\bar{x} \in X$ such that for each $y \in X$, there exists $s \in T \bar{x}$ such that

$$
\psi(s, z, y) \notin-\operatorname{int} C \bar{x} \text { for all } z \in G \bar{x} \text {. }
$$

For various special cases of (IVVI), readers can refer to [13]. As far as (IVVI)' is concerned, (IVVI) ${ }^{\prime}$ becomes the VVI studied by Lee et al. [10] provided $\psi(s, x, y)=$ $\langle s, y-x\rangle$ where $\langle s, y-x\rangle$ denotes the evaluation of $s$ at $y-x$. 
In fact (IVVI) ${ }^{\prime}$ can be viewed as an extension of (IVVI) because the former reduces to the latter by putting $G x=\{x\}$ for all $x \in X$. Now we give the generalized pseudomonotonicity and the generalized hemicontinuity on the multifunction T. $T$ is said to be

(i) generalized C-pseudomonotone w.r.t. $\psi$ if for any $x, y \in X, \exists s \in T x$ such that

$$
\psi(s, x, y) \notin-\operatorname{int} C x \text { implies } \forall t \in T y,-\psi(t, y, x) \notin-\operatorname{int} C x ;
$$

(ii ) generalized weakly C-pseudomonotone w.r.t. $\psi$ if for any $x, y \in X, \exists s \in T x$ such that

$$
\psi(s, x, y) \notin-\operatorname{int} C x \text { implies } \exists t \in T y,-\psi(t, y, x) \notin-\operatorname{int} C x \text {; and }
$$

(iii) generalized hemicontinuous w.r.t. $\psi$ if for any $x, y \in X$ and $\alpha \in[0,1]$, the multifunction

$$
\alpha \mapsto \psi(T(x+\alpha(y-x)), x, y)
$$

is upper semicontinuous at $0^{+}$, where $\psi(T(x+\alpha(y-x)), x, y)=\{\psi(t, x, y) \mid$ $t \in T(x+\alpha(y-x))\}$.

Also $T$ is said to be

(i )' generalized C-pseudomonotone if for any $x, y \in X, \exists s \in T x$ such that

$$
\langle s, y-x\rangle \notin-\operatorname{int} C x \text { implies } \forall t \in T y,\langle t, y-x\rangle \notin-\operatorname{int} C x \text {; and }
$$

(ii) ${ }^{\prime}$ generalized weakly $C$-pseudomonotone if for any $x, y \in X, \exists s \in T x$ such that

$$
\langle s, y-x\rangle \notin-\operatorname{int} C x \text { implies } \exists t \in T y,\langle t, y-x\rangle \notin-\operatorname{int} C x \text {; and }
$$

(iii) $^{\prime}$ generalized hemicontinuous if for any $x, y \in X$ and $\alpha \in[0,1]$, the multifunction

$$
\alpha \mapsto\langle T(x+\alpha(y-x)), y-x\rangle
$$

is upper semicontinuous at $0^{+}$, where $\langle T(x+\alpha(y-x)), y-x\rangle=\{\langle s, y-x\rangle \mid$ $s \in T(x+\alpha(y-x))\}$.

The following lemma is immediate from the above definitions.

Lemma 2.1. Let $E, X, F, C, P, \psi$, and $T$ be the same as in the above definitions. Then we have

(i) $T$ is generalized C-pseudomonotone w.r.t. $\psi \Rightarrow T$ is generalized weakly C-pseudomonotone w.r.t. $\psi$; and 
(ii) $T$ is generalized hemicontinuous $\Rightarrow T$ is generalized hemicontinuous w.r.t. $\psi(s, x, y)=\langle s, y-x\rangle$.

Example 2.1. Let $E=X=F=R, C x=[0, \infty)$ for all $x \in R$ and $\psi(s, x, y)=s(y-x)$ for any $s, x, y \in R$. Define a multifunction $T: R \rightarrow 2^{R}$ by for any $x \in R, T x=[x, \infty)$. Then $T$ is generalized weakly $C$-pseudomonotone (w.r.t. $\psi$ ), but $T$ is not generalized $C$-pseudomonotone (w.r.t. $\psi$ ). Moreover, $T$ is generalized hemicontinuous (w.r.t. $\psi$ ).

The following particular form of Park [15, Theorem 5] is a basic machinary to derive main results.

Theorem 2.1. Let $X$ be a nonempty convex subset of a real Hausdorff topological vector space $E, K$ a nonempty compact subset of $X$. Let $A, B: X \rightarrow 2^{X}$ be two multifunctions. Suppose that

(i) for each $x \in X, A x \subset B x$;

(ii) for each $x \in X, B x$ is convex;

(iii) for each $x \in K, A x$ is nonempty;

(iv) for each $y \in X, A^{-1} y$ is open in $X$; and

(v) for each finite subset $N$ of $X$, there exists a nonempty compact convex subset $L_{N}$ of $X$ containing $N$ such that for each $x \in L_{N} \backslash K, A x \cap L_{N} \neq \emptyset$. Then $B$ has a fixed point $x_{0}$; that is, $x_{0} \in B x_{0}$.

\section{IVVI Under Generalized PSEUdomonotonicity}

We provide the first main result concerned with the existence of solutions of (IVVI) under the generalized pseudomonotonicity condition.

Theorem 3.1. Let $E$ be a real Hausdorff topological vector space on which the topological dual space $E^{*}$ of $E$ separates points, $X$ a nonempty convex subset of $E, F$ another real Hausdorff topological vector space on which the topological dual space $F^{*}$ of $F$ separates points, and $C: X \rightarrow 2^{F}$ a multifunction such that for each $x \in X, C x$ is a convex cone in $F$ with $-\operatorname{int} C x \neq \emptyset$ and $C x \neq F$. Let $P:=\bigcap_{x \in X} C x, \psi: L(E, F) \times X \times X \rightarrow F$ be a function, and $T: X \rightarrow 2^{L(E, F)} a$ multifunction. Let $K$ be a nonempty weakly compact subset of $X$ and $W: X \rightarrow 2^{F}$, $W x=F \backslash(-\operatorname{int} C x)$, such that the graph $G r(W)$ of $W$ is weakly closed in $X \times F$. Assume that the following conditions are satisfied;

( i ) $T$ is generalized C-pseudomonotone w.r.t. $\psi$;

(ii) $T$ is generalized hemicontinuous w.r.t. $\psi$; 
(iii) for each $s \in L(E, F)$ and $x \in X, \psi(s, x, \cdot)$ is $P$-convex, that is, for any $y, z \in X$ and $\alpha \in[0,1]$,

$$
\psi(s, x, \alpha y+(1-\alpha) z) \in \alpha \psi(s, x, y)+(1-\alpha) \psi(s, x, z)-P
$$

(iv) for each $t \in L(E, F)$ and $x \in X, \psi(t, x, \cdot)$ is continuous where both $X$ and $F$ are endowed with the weak topologies;

(v) for any $x \in X$ and $s \in T x, \psi(s, x, x) \in P$;

(vi) for any $s \in L(E, F), x, y \in X$ and $\alpha \in[0,1], \psi(s, x+\alpha(y-x), y)=$ $(1-\alpha) \psi(s, x, y) ;$ and

(vii) for each finite subset $N$ of $X$, there exists a nonempty weakly compact convex subset $L_{N}$ of $X$ containing $N$ such that for each $x \in L_{N} \backslash K$, there exists $y \in L_{N}$ satisfying $-\psi(t, y, x) \in-\operatorname{int} C x$ for some $t \in T y$.

Then there exists $\bar{x} \in K$ such that $\bar{x}$ is a solution of (IVVI).

Proof. Let $X$ be equipped with the weak topology from $E$. Define two multifunctions $A, B: X \rightarrow 2^{X}$ to be

$$
\begin{aligned}
& A x:=\{y \in X \mid \exists t \in T y \text { such that }-\psi(t, y, x) \in-\operatorname{int} C x\} \\
& B x:=\{y \in X \mid \forall s \in T x, \psi(s, x, y) \in-\operatorname{int} C x\} .
\end{aligned}
$$

The proof is organized in the following parts.

(a) Since $T$ is generalized $C$-pseudomonotone w.r.t. $\psi$, for any $x \in X, A x \subset B x$.

(b) For each $x \in X, B x$ is convex. Indeed, when $y, z \in B x$ and $\alpha \in[0,1]$, we have for any $s \in T x$,

$$
\begin{aligned}
\psi(s, x, \alpha y+(1-\alpha) z) & \in \alpha \psi(s, x, y)+(1-\alpha) \psi(s, x, z)-P \\
& \subset \alpha(-\operatorname{int} C x)+(1-\alpha)(-\operatorname{int} C x)-P \\
& \subset-\operatorname{int} C x-C x=-\operatorname{int} C x .
\end{aligned}
$$

Hence $\alpha y+(1-\alpha) z \in B x$, as desired.

(c) For each $y \in X, A^{-1} y$ is open in $X$. In fact, let $\left\{x_{\lambda}\right\}$ be a net in $\left(A^{-1} y\right)^{c}$ weakly convergent to $x \in X$. Then $y \notin A x_{\lambda}$ and hence for any $t \in T y,-\psi\left(t, y, x_{\lambda}\right) \notin-\operatorname{int} C x_{\lambda}$. Thus for any $t \in T y,-\psi\left(t, y, x_{\lambda}\right) \in W x_{\lambda}$. Since $\left(x_{\lambda},-\psi\left(t, y, x_{\lambda}\right)\right) \in G r(W)$, by virtue of assumption (iv) and the weak closedness of $\operatorname{Gr}(W),-\psi(t, y, x) \in W x$ for any $t \in T y$, that is, for any $t \in T y,-\psi(t, y, x) \notin-\operatorname{int} C x$, and hence $y \notin A x$, namely, $x \in\left(A^{-1} y\right)^{c}$. Therefore $\left(A^{-1} y\right)^{c}$ is closed in $X$, whence $A^{-1} y$ is open in $X$. 
(d) By hypothesis (vii), for each finite subset $N$ of $X$, there exists a nonempty weakly compact convex subset $L_{N}$ of $X$ containing $N$ such that for each $x \in L_{N} \backslash K$, there exists $y \in L_{N}$ satisfying $-\psi(t, y, x) \in-\operatorname{int} C x$ for some $t \in T y$. Thus for each $x \in L_{N} \backslash K$, there exists $y \in L_{N}$ such that $y \in A x$, hence $L_{N} \cap A x \neq \emptyset$.

(e) $B$ has no fixed point. If not, there exists $x \in X$ such that for any $s \in T x$, $\psi(s, x, x) \in-\operatorname{int} C x$. By assumption (v), for any $s \in T x, \psi(s, x, x) \in$ - int $C x \cap C x=\emptyset$, which is a contradiction.

(f) From (a)-(e), we see, by Theorem 2.1, that there must be $\bar{x} \in K$ such that $A \bar{x}=\emptyset$, namely, for any $y \in X, y \notin A \bar{x}$, that is, for any $y \in X$ and $t \in T y$,

$$
-\psi(t, y, \bar{x}) \notin-\operatorname{int} C \bar{x} \text {. }
$$

We claim that $\bar{x}$ is a solution of (IVVI). Suppose to the contrary that $\bar{x}$ is not a solution of (IVVI). Then there eixsts $\bar{y} \in X$ such that for any $s \in T \bar{x}$,

$$
\psi(s, \bar{x}, \bar{y}) \in-\operatorname{int} C \bar{x} .
$$

Let $x_{\alpha}:=\bar{x}+\alpha(\bar{y}-\bar{x})$ for $\alpha \in[0,1]$. Since $X$ is convex, $x_{\alpha} \in X$. Define a multifunction $H:[0,1] \rightarrow 2^{F}$ by for any $\alpha \in[0,1], H(\alpha):=\{\psi(s, \bar{x}, \bar{y}) \mid s \in$ $T x_{\alpha}$ \}. Then by (2), $H(0) \subset-\operatorname{int} C \bar{x}$. Since $T$ is generalized hemicontinuous w.r.t. $\psi$, there exists $\hat{\alpha} \in(0,1]$ such that for any $\alpha \in[0, \hat{\alpha}), H(\alpha) \subset-\operatorname{int} C \bar{x}$. Hence for any $\alpha \in(0, \hat{\alpha})$ and $s \in T x_{\alpha}$,

$$
\psi(s, \bar{x}, \bar{y}) \in-\operatorname{int} C \bar{x} .
$$

Fix $\alpha \in(0, \hat{\alpha})$. By the $P$-convexity of $\psi\left(s, x_{\alpha}, \cdot\right)$, we have for any $s \in T x_{\alpha}$,

$$
\begin{aligned}
\psi\left(s, x_{\alpha}, x_{\alpha}\right) & =\psi\left(s, x_{\alpha}, \alpha \bar{y}+(1-\alpha) \bar{x}\right) \\
& \in \alpha \psi\left(s, x_{\alpha}, \bar{y}\right)+(1-\alpha) \psi\left(s, x_{\alpha}, \bar{x}\right)-P .
\end{aligned}
$$

From (3) and assumptions (v) and (vi), we have for any $s \in T x_{\alpha}$,

$$
\begin{aligned}
-(1-\alpha) \psi\left(s, x_{\alpha}, \bar{x}\right) & \in \alpha \psi\left(s, x_{\alpha}, \bar{y}\right)-\psi\left(s, x_{\alpha}, x_{\alpha}\right)-P \\
& \subset \alpha(1-\alpha) \psi(s, \bar{x}, \bar{y})-P-P \\
& \subset-\operatorname{int} C \bar{x}-C \bar{x}-C \bar{x} \\
& \subset-\operatorname{int} C \bar{x} .
\end{aligned}
$$

Thus for any $s \in T x_{\alpha},-\psi\left(s, x_{\alpha}, \bar{x}\right) \in-\operatorname{int} C \bar{x}$, which contradicts (1). This completes the proof.

Corollary 3.1. Let $E, F, X, K, C, W, G r(W)$ and $P$ be the same as in Theorem 3.1. Let $T: X \rightarrow 2^{L(E, F)}$ be a multifunction. Assume that the following conditions are satisfied: 
( i ) $T$ is generalized C-pseudomonotone;

(ii ) $T$ is generalized hemicontinuous; and

(iii) for each finite subset $N$ of $X$, there exists a nonempty weakly compact convex subset $L_{N}$ of $X$ containing $N$ such that for each $x \in L_{N} \backslash K$, there exists $y \in L_{N}$ satisfying $\langle t, y-x\rangle \in-\operatorname{int} C x$ for some $t \in T y$.

Then there exists $\bar{x} \in K$ such that

$$
\forall y \in X, \exists s \in T \bar{x}: \quad\langle s, y-\bar{x}\rangle \notin-\operatorname{int} C \bar{x} .
$$

Proof. Taking $\psi(s, x, y)=\langle s, y-x\rangle$ in Theorem 3.1, we get the result. Indeed, it is straightforward to check the conditions (i)-(vii) of Theorem 3.1 except the continuity of $s:(E$, weak $) \rightarrow(F$, weak $)$. But this directly follows from the definition of the weak topologies for $E$ and $F$. (See Kelley and Namioka [5, 16.1 (iv) p.140]).

Remark 3.1. Corollary 3.1 is a noncompact version of Konnov and Yao [6, Theorem 3.1] in the context of a Hausdorff t.v.s. instead of a Banach space.

Example 3.1. Let $E=F=R, X=[0, \infty)$ and $K=[0,1]$. Let $T x=$ $\left[x^{2}+1, \infty\right)$ and $C x=[0, \infty)$ for any $x \in X$, and $\psi(s, x, y)=s(y-x)$ for any $s \in R$ and $x, y \in X$. For each finite subset $N$ of $X$, we take $L_{N}:=\operatorname{co} N$, where $\operatorname{co} N$ denotes the convex hull of $N$. Then all the assumptions of Theorem 3.1 are satisfied. Moreover, $0 \in K$ is a solution of (IVVI).

It is necessary to adopt more definitions in Konnov and Yao [6]. A point $\bar{x} \in X$ is said to be a strong solution of (IVVI) if there exists $\bar{t} \in T \bar{x}$ such that

$$
\psi(\bar{t}, \bar{x}, y) \notin-\operatorname{int} C \bar{x} \quad \text { for all } \quad y \in X .
$$

For $s \in F^{*}$, we set

$$
H(s)=\{z \in F \mid\langle s, z\rangle \geq 0\} .
$$

Then $T: X \rightarrow 2^{L(E, F)}$ is said to be

(i ) $H(s)$-pseudomonotone w.r.t. $\psi$ if for any $x, y \in X$ and for every $t^{\prime} \in T x$, $t^{\prime \prime} \in T y$ we have

$$
\psi\left(t^{\prime}, x, y\right) \in H(s) \text { implies }-\psi\left(t^{\prime \prime}, y, x\right) \in H(s) ; \text { and }
$$

(ii) $H(s)$-pseudomonotone if for any $x, y \in X$ and for every $t^{\prime} \in T x, t^{\prime \prime} \in T y$, we have 


$$
\left\langle t^{\prime}, y-x\right\rangle \in H(s) \text { implies }\left\langle t^{\prime \prime}, y-x\right\rangle \in H(s) .
$$

By a scalization method we derive the existence of a strong solution of (IVVI) under an appropriate condition.

Theorem 3.2. Let $E, \psi, C$ and $P$ be the same as in Theorem 3.1, and $X$ be a nonempty weakly compact convex subset of E. Let $F$ be a Hausdorff l.c.s. and $L(E, F)$ be equipped with either the topology of pointwise convergence or the topology of bounded convergence, and $F^{*}$ the topological dual space of $F$. Let $T: X \rightarrow 2^{L(E, F)}$ be a multifunction. Assume that $C_{+}^{*} \backslash\{0\} \neq \emptyset$, where $C_{+}=c o\left(\cup_{x \in X} C x\right)$ and

$$
C_{+}^{*}=\left\{w \in F^{*} \mid\langle w, y\rangle \geq 0 \text { for any } y \in C_{+}\right\} .
$$

Assume further that the following conditions are satisfied:

(i ) Let $s \in C_{+}^{*} \backslash\{0\}$ and $H(s) \neq F$. T is $H(s)$-pseudomonotone w.r.t. $\psi$;

(ii) $T$ is generalized hemicontinuous w.r.t. $\psi$ and for any $x \in X$, Tx is nonempty;

(iii) for each $t \in L(E, F)$ and $x \in X, \psi(t, x, \cdot)$ is $P$-convex and continuous where both $X$ and $F$ are endowed with the weak topologies;

(iv) for each $x, y \in X,-\psi(\cdot, x, y)$ is $P$-convex and continuous where $F$ is endowed with the weak topology;

(v) for any $x \in X$ and $t \in T x, \psi(t, x, x) \in P$; and

(vi) for any $t \in L(E, F), x, y \in X$ and $\alpha \in[0,1], \psi(t, x+\alpha(y-x), y)=$ $(1-\alpha) \psi(t, x, y)$.

Then :

(I) There exists a solution $\bar{x} \in X$ of (IVVI).

(II) If, for each $x \in X, T x$ is convex and compact, there exists a strong solution of (IVVI).

Proof. (I) For notational simplicity, we define a mapping $\psi_{s}: L(E, F) \times X \times$ $X \rightarrow R$ by $\psi_{s}(t, x, y)=s(\psi(t, x, y))$. Due to the $H(s)$-pseudomonotonicity of $T$ w.r.t. $\psi$, for every pair of $x, y \in X$ and for all $t^{\prime} \in T x, t^{\prime \prime} \in T y$, we have

$$
\psi_{s}\left(t^{\prime}, x, y\right) \geq 0 \text { implies }-\psi_{s}\left(t^{\prime \prime}, y, x\right) \geq 0 .
$$

Since $T$ is generalized hemicontinuous w.r.t. $\psi$, for any $x, y \in X$ and $\alpha \in[0,1]$,

$$
\alpha \mapsto \psi_{s}(T(x+\alpha(y-x)), x, y)
$$

is upper semicontinuous at $0^{+}$. Moreover, $\psi_{s}(t, x, \cdot)$ is convex and continuous by virtue of (iii). In addtion, $\psi_{s}(t, x, x) \geq 0$ for any $x \in X$ and $t \in T x$ by (v). 
Let $X$ be equipped with the weak topology from $E$. Define two multifunctions $A, B: X \rightarrow 2^{X}$ to be

$$
\begin{aligned}
& A x:=\left\{y \in X \mid \exists t \in T y \text { such that }-\psi_{s}(t, y, x)<0\right\} \\
& B x:=\left\{y \in X \mid \forall v \in T x \text { such that } \psi_{s}(v, x, y)<0\right\} .
\end{aligned}
$$

Then we can easily check the follwings:

(a) for any $x \in X, A x \subset B x$;

(b) for any $x \in X, B x$ is convex;

(c) for each $y \in X, A^{-1} y$ is open in $X$; and

(d) $B$ has no fixed point. So, by Theorem 2.1 , there exists $\bar{x} \in K$ such that $A \bar{x}=\emptyset$. Thus, for each $y \in X$ and $t \in T y,-\psi_{s}(t, y, \bar{x}) \geq 0$. By a similar argument to the second part of the proof of Theorem 3.1, we can show that

$$
\forall y \in X, \exists \bar{t} \in T \bar{x} \text { such that } \psi_{s}(\bar{t}, \bar{x}, y) \geq 0 .
$$

Note that int $H(s)=s^{-1}(0, \infty)$. For a proof, see Lee and Kum [13, Theorem 4.1]. From this observation and the fact that $-\operatorname{int} C \bar{x} \subset-\operatorname{int} H(s)$, we can conclude that

$$
\forall y \in X, \exists \bar{t} \in T \bar{x} \text { such that } \psi(\bar{t}, \bar{x}, y) \notin-\operatorname{int} C \bar{x} \text {. }
$$

(II) Suppose that $T \bar{x}$ is convex and compact. By (4), (iv) and Kneser's minimax theorem [7], we have

$$
\max _{t \in T \bar{x}} \min _{y \in X} \psi_{s}(t, \bar{x}, y)=\min _{y \in X} \max _{t \in T \bar{x}} \psi_{s}(t, \bar{x}, y) \geq 0 .
$$

Thus there exists $\bar{t} \in T \bar{x}$ such that

$$
\psi_{s}(\bar{t}, \bar{x}, y) \geq 0 \text { for all } y \in X .
$$

Recalling int $H(s)=s^{-1}(0, \infty)$ yields

$$
\psi(\bar{t}, \bar{x}, y) \notin-\operatorname{int} C \bar{x} \text { for all } y \in X,
$$

which implies that $\bar{x}$ is a strong solution of (IVVI).

Corollary 3.2. Let $E, F, X, C, C_{+}, C_{+}^{*}$ and $P$ be the same as in Theorem 3.2 , and $L(E, F)$ be equipped with the topology of pointwise convergence. Let $T: X \rightarrow 2^{L(E, F)}$ be a multifunction. Assume that the following conditions are satisfied:

(i) Let $s \in C_{+}^{*} \backslash\{0\}$ and $H(s) \neq F$. $T$ is $H(s)$-pseudomonotone; and 
(ii) $T$ is generalized hemicontinuous and for any $x \in X, T x$ is nonempty.

Then:

(I) There exists $\bar{x} \in X$ satisfying

$$
\forall y \in X, \exists \bar{t} \in T \bar{x} \text { such that }\langle\bar{t}, y-\bar{x}\rangle \notin-\operatorname{int} C \bar{x} .
$$

(II) If, for each $x \in X, T x$ is convex and compact, there exists $\bar{x} \in X$ and $\bar{t} \in T \bar{x}$ such that

$$
\langle\bar{t}, y-\bar{x}\rangle \notin-\operatorname{int} C \bar{x} \text { for all } y \in X .
$$

Proof. Taking $\psi(s, x, y)=\langle s, y-x\rangle$ in Theorem 3.2, we get the result.

Remark 3.2. Corollary 3.2 is a generalized version of Konnov and Yao [6, Theorem 4.1] in the context of a Hausdorff locally convex space.

\section{4. (IVVI)' Without Generalized PSEUdomonotonicity}

Now we are in a position to introduce the following existence theorem of (IVVI)' for compact-valued and upper semicontinuous multifunctions without the generalized pseudomonotonicity condition. Using this result, we will deduce the final main result of this paper, say, Theorem 4.2.

Theorem 4.1. Let $E, F, X, K, C, W, G r(W)$ and $P$ be the same as in Theorem 3.1. Suppose that $L(E, F)$ is equipped with either the topology of pointwise convergence or the topology of bounded convergence. Let $\psi: L(E, F) \times$ $X \times X \rightarrow F$ be a function, $T: X \rightarrow 2^{L(E, F)}$ a multifunction, and $G: X \rightarrow 2^{X} a$ multifunction. Assume that the following conditions are satisfied:

(i ) $T$ is compat-valued and upper semicontinuous where $X$ is equipped with the weak topology, and $T(X)$ is contained in a compact subset of $L(E, F)$;

(ii) for each $s \in L(E, F), \psi(s, \cdot, \cdot)$ is $P$-convex with respect to two variables, that is, for any $y_{1}, y_{2}, z_{1}, z_{2} \in X$ and $\alpha \in[0,1]$,

$$
\begin{aligned}
& \psi\left(s, \alpha z_{1}+(1-\alpha) z_{2}, \alpha y_{1}+(1-\alpha) y_{2}\right) \in \alpha \psi\left(s, z_{1}, y_{1}\right) \\
& \quad+(1-\alpha) \psi\left(s, z_{2}, y_{2}\right)-P
\end{aligned}
$$

(iii) for each $y \in X, \psi(\cdot, \cdot, y)$ is continuous where both $X$ and $F$ are endowed with the weak topologies;

(iv) $G$ is convex-valued and lower semicontinuous on $X$ with the weak topology;

(v) for each $x \in X, \exists s \in T x$ such that $\psi(s, z, x) \notin-$ intC $x$ for all $z \in G x$; and 
(vi) for each finite subset $N$ of $X$, there exists a nonempty weakly compact convex subset $L_{N}$ of $X$ containing $N$ such that for each $x \in L_{N} \backslash K$, there exists $y \in L_{N}$ such that for any $s \in T x, \psi(s, z, y) \in-\operatorname{int} C x$ for some $z \in G x$.

Then there exists $\bar{x} \in K$ such that $\bar{x}$ is a solution of (IVVI)'.

Proof. Let $X$ be equipped with the weak topology from $E$. Define a multifunction $A: X \rightarrow 2^{X}$ to be

$$
A x:=\{y \in X \mid \forall s \in T x, \exists z \in G x \quad \text { s.t. } \quad \psi(s, z, y) \in-\operatorname{int} C x\} .
$$

The proof is appealing to a similar argument in that of Theorem 3.1.

(a) For each $x \in X, A x$ is convex. This follows directly from the assumption (ii) and the convexity of $G x$.

(b) For each $y \in X, A^{-1} y$ is open in $X$. In fact, let $\left\{x_{\lambda}\right\}$ be a net in $\left(A^{-1} y\right)^{c}$ weakly convergent to $x \in X$. Then $y \notin A x_{\lambda}$ and hence there exists $s_{\lambda} \in T x_{\lambda}$ such that for any $z \in G x_{\lambda}$,

$$
\psi\left(s_{\lambda}, z, y\right) \notin-\operatorname{int} C x_{\lambda} .
$$

Since $T(X)$ is contained in a compact subset of $L(E, F)$, we may assume that $s_{\lambda}$ converges to $s \in L(E, F)$. Since $T$ is compact-valued and upper semicontinuous, the graph of $T$ is closed, and so $s \in T x$. Since $G$ is lower semicontinuous, for any $w \in G x$, there exists $z_{\lambda} \in G x_{\lambda}$ weakly convergent to $w$. Since $\left(x_{\lambda}, \psi\left(s_{\lambda}, z_{\lambda}, y\right)\right) \in G r(W)$, by virtue of (iii) and the weak closedness of $G r(W)$, we have $(x, \psi(s, w, y)) \in G r(W)$. Hence $\psi(s, w, y) \notin$ -int $C x$ for any $w \in G x$, that is, $x \in\left(A^{-1} y\right)^{c}$. Therefore $\left(A^{-1} y\right)^{c}$ is closed in $X$, so $A^{-1} y$ is open in $X$.

(c) By (vi), for each finite subset $N$ of $X$, there exists a nonempty weakly compact convex subset $L_{N}$ of $X$ containing $N$ such that for each $x \in L_{N} \backslash K$, there exists $y \in L_{N}$ such that for any $s \in T x, \psi(s, z, y) \in-\operatorname{int} C x$ for some $z \in G x$. Thus $L_{N} \cap A x \neq \emptyset$.

(d) $A$ has no fixed point by (v). From (a) - (d), we see, by Theorem 2.1, that there must be an $\bar{x} \in K$ such that $A \bar{x}=\emptyset$, namely, for each $y \in X$, there exists $s \in T \bar{x}$ such that

$$
\psi(s, z, y) \notin-\operatorname{int} C \bar{x} \text { for any } z \in G \bar{x} .
$$

This completes the proof.

Corollary 4.1. (cf. Lee et al. [10, Theorem 2.1]) Let E be a Hausdorff l.c.s. and $X$ a weakly compact convex subset of $E$. Let $F, C, W, G r(W)$ and $P$ be the same as in Theorem 3.1. Suppose that $L(E, F)$ is equipped with the topology of bounded convergence. Let $T: X \rightarrow 2^{L(E, F)}$ be a multifunction and $G: X \rightarrow 2^{X}$ a multifunction. Assume that the following conditions are satisfied: 
(i ) $T$ is compat-valued and upper semicontinuous where $X$ is equipped with the weak topology;

(ii) $G$ is convex-valued and lower semicontinuous on $X$ with the weak topology; and

(iii) for each $x \in X, \exists s \in T x$ such that $\langle s, x-z\rangle \notin-\operatorname{int} C x$ for all $z \in G x$.

Then there exists $\bar{x} \in X$ such that for each $y \in X$, there exists $s \in T \bar{x}$ satisfying

$$
\langle s, y-z\rangle \notin-\operatorname{int} C \bar{x} \text { for any } z \in G \bar{x} \text {. }
$$

Proof. Put $\psi(s, x, y)=\langle s, y-x\rangle$ and $X=L_{N}=K$ in Theorem 3.2. Recall that $X$ is strongly bounded because $E$ is a Hausdorff 1.c.s. The image $T(X)$ is obviously compact since $T$ is compact-valued and upper semicontinuous. Appealing to Lemma 2.4 in Lee and Kum [13], we can easily check that all the conditions in Theorem 4.1 are satisfied. Therefore we get the conclusion.

Using the selection theorem of Yannelis and Prabhakar [17] and Theorem 4.1 we prove the following existence theorem of (IVVI) ${ }^{\prime}$ for multifunctions with convex values and open lower sections without the generalized pseudomonotonicity condition.

Theorem 4.2. Let $E, F, X, C, W, G r(W)$ and $P$ be the same as in Theorem 3.1 except that $X$ is a nonempty weakly compact convex set. Let $L(E, F)$ be equipped with either the topology of pointwise convergence or the topology of bounded convergence. Assume that the following conditions are satisfied:

(i ) $T$ is a nonempty convex-valued multifunction such that for any $s \in L(E, F)$, $T^{-1}(s)$ is weakly open in $X$;

(ii) for each $s \in L(E, F), \psi(s, \cdot, \cdot)$ is $P$-convex with respect to two variables;

(iii) for each $y \in X, \psi(\cdot, \cdot, y)$ is continuous where both $X$ and $F$ are endowed with the weak topologies;

(iv) $G$ is convex-valued and lower semicontinuous on $X$ with the weak topology; and

(v) for each $x \in X, s \in T x$, and $z \in G x, \psi(s, z, x) \notin-\operatorname{int} C x$.

Then there exists $\bar{x} \in X$ and $s \in T \bar{x}$ such that

$$
\psi(s, z, y) \notin-\operatorname{int} C \bar{x} \text { for any } y \in X \text { and } z \in G \bar{x} \text {. }
$$

Proof. Let $X$ be equipped with the weak topology from $E$. Since $T$ has open lower sections, that is, for any $t \in L(E, F), T^{-1}(t):=\{x \in X \mid t \in T x\}$ is open 
in $X$, and $T$ has nonempty convex values, by the selection theorem of Yannelis and Prabhakar [17], there exists a continuous function $f: X \rightarrow L(E, F)$ such that $f x \in T x$ for any $x \in X$. By Theorem 3.2, there exists $\bar{x} \in X$ such that

$$
\psi(f \bar{x}, z, y) \notin-\operatorname{int} C \bar{x} \text { for any } y \in X \text { and } z \in G \bar{x} .
$$

Putting $s=f \bar{x} \in T \bar{x}$, we obtain the conclusion of Theorem 4.2.

Corollary 4.2. (cf. Lee et al. [10, Corollary 2.3]) Let E, F, X, C, W, Gr $(W)$ and $P$ be the same as in Corollary 4.1. Let $T: X \rightarrow 2^{L(E, F)}$ be a multifunction and $G: X \rightarrow 2^{X}$ a multifunction. Assume that the following conditions are satisfied:

(i ) $T$ is a nonempty convex-valued multifunction such that for any $s \in L(E, F)$, $T^{-1}(s)$ is weakly open in $X$;

(ii ) $G$ is convex-valued and lower semicontinuous on $X$ with the weak topology; and

(iii) for each $x \in X, s \in T x$, and $z \in G x,\langle s, x-z\rangle \notin-\operatorname{int} C x$.

Then there exists $\bar{x} \in X$ and $s \in T \bar{x}$ such that

$$
\langle s, y-z\rangle \notin-\operatorname{int} C \bar{x} \text { for any } y \in X \text { and } z \in G \bar{x} \text {. }
$$

Proof. Taking $\psi(s, x, y)=\langle s, y-x\rangle$ in Theorem 4.2, we obtain the result as in Corollary 4.1.

\section{REFERENCES}

1. Q. H. Ansari, Extended generalized vector variational-like inequalities, Ann. Sci. Math. Quebec 21 (1997), 1-11.

2. Q. H. Ansari and J. C. Yao, On strong solutions of the generalized implicit vector variational problem, Adv. Nonlinear Var. Inequal. 2 (1999), 1-10.

3. G. Y. Chen, Existence of solutions for a vector variational inequality: an extension of the Hartman-Stampacchia theorem, J. Optim. Theory Appl. 74 (1992), 445-456.

4. F. Giannessi, Therorems of alternative, quadratic programs and complementarity problems, in: Variational Inequalities and Complementarity Problems (R. W. Cottle, F. Giannessi and J. L. Lions, Eds.), John Wiley and Sons, Chichester, England, 1980, pp. 151-186.

5. J. L. Kelly and I. Namioka, Linear Topological Spaces, Springer-Verlag, Berlin/New York, 1963.

6. I. V. Konnov and J. C. Yao, On the generalized vector variational inequality problem, J. Math. Anal. Appl. 206 (1997), 42-58. 
7. H. Kneser, Sur un Théoreme Fondamantal de la Théorie des Jeux, C. R. Acad. Sci. Paris 234 (1952), 2418-2420.

8. T. C. Lai and J. C. Yao, Existence results for VVIP, Appl. Math. Lett. 9 (1996), 17-19.

9. B. S. Lee, G. M. Lee and D. S. Kim, Generalized vector variational-like inequalities on locally convex Hausdorff topological vector spaces, Indian J. Pure Appl. Math. 28 (1997), 33-41.

10. G. M. Lee, B. S. Lee, D. S. Kim and G. Y. Chen, On vector variational inequalities for multifunctions, Indian J. Pure Appl. Math. 28 (1997), 633-639.

11. G. M. Lee, D. S. Kim, B. S. Lee and N. D. Yen, Vector variational inequality as a tool for studying vector optimization problems, Nonlinear Anal. 34 (1998), 745-765.

12. G. M. Lee and S. H. Kum, Vector variational inequalities in a Hausdorff topological vector space, in: Vector Variational Inequalities and Vector Equilibria (F. Giannessi, Ed.), Kluwer Academic Publishers, Dordrecht, The Netherlands, 2000, pp. 307-320.

13. G. M. Lee and S. H. Kum, On implicit vector variational inequalities, J. Optim. Theory Appl. 104 (2000), 409-425.

14. K. L. Lin, D. P. Yang and J. C. Yao, Generalized vector variational inequalities, $J$. Optim. Theory Appl. 92 (1997), 117-125.

15. S. Park, Foundations of the KKM theory via coincidences of composites of upper semicontinuous maps, J. Korean Math. Soc. 31 (1994), 493-519.

16. X. Q. Yang, Vector variational inequality and its duality, Nonlinear Anal. 21 (1993), 869-877.

17. N. C. Yannelis and N. D. Prabhakar, Existence of maximal elements and equilibria in linear topological spaces, J. Math. Economics 12 (1983), 233-245.

18. S. J. Yu and J. C. Yao, On vector variational inequalities, J. Optim. Theory Appl. 89 (1996), 749-769.

Sangho Kum

Department of Mathematics Education

Chungbuk National University

Cheongju 361-763, Korea

Gue Myung Lee

Department of Applied Mathematics

Pukyong National University

Pusan 608-737, Korea 